\title{
An Unusual Location of Deep Venous Thrombosis Associated with Ischemic Stroke and Persistent Foramen Ovale
}

\author{
J. Erharhaghen ${ }^{a} \quad$ M. Bartz ${ }^{a} \quad$ S. Di Giovanni ${ }^{a} \quad$ A. Melms ${ }^{a}$ \\ T. Haarmeier ${ }^{\mathrm{a}}$ L. Sieverding ${ }^{\mathrm{b}}$

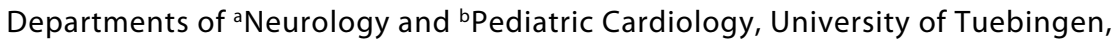 \\ Tuebingen, Germany
}

\section{Key Words}

Deep venous thrombosis $\cdot$ Persistent foramen ovale $\cdot$ Cryptogenic stroke $\cdot$ Ischemic stroke

\begin{abstract}
Up to $40 \%$ of ischemic strokes have no known cause (cryptogenic). The prevalence of persistent foramen ovale (PFO) amongst patients with cryptogenic stroke (CS) is twice as high as that of the normal population, therefore suggesting a causal relationship between the two entities. However, PFO by itself is not sufficient to cause stroke, as an embolic source is needed. This source is often unknown, making the causal relationship between CS and PFO hard to demonstrate. The most frequent, although still seldom, identifiable cause of embolism in an otherwise cryptogenic stroke associated with PFO is a deep venous thrombosis (DVT) of the lower extremities. Here, we present a unique case of brachiocephalic venous DVT associated with PFO and ischemic stroke in a young patient. As the search for DVT in patients with PFO and stroke is often limited to the lower extremities, this case may suggest that an unspecified number of DVTs are overlooked. Our report lends support to paradoxical embolism as a mechanism of stroke in patients with PFO and does, at least in selected cases, suggest a more detailed search for DVT beyond the lower extremities.
\end{abstract}

\section{Introduction}

We report the unique case of a patient with brachiocephalic deep venous thrombosis (DVT) and persistent foramen ovale (PFO) who developed ischemic stroke in the absence of other risk factors for stroke. This may suggest the need for more detailed search for the embolic source in selected cases. 
A large percentage of ischemic strokes have no known cause (up to 40\%) [1,2]. PFO prevalence amongst cases of cryptogenic stroke (CS) is twice as high as that of the normal population [3], making a causal relationship possible. Stroke mechanism in this setting is thought to be a paradoxical embolism arising from a venous or right atrial source. The embolic source is, however, only seldom found [4], possibly because the search for thrombotic episodes is often restricted to the lower extremities, which makes up for only approximately one third of the embolic sources in clinically plausible cases of paradoxical embolism and stroke [5]. In our patient, the thrombus was detected using brachiocephalic venography done during PFO closure.

\section{Case Report}

A 40-year-old mildly overweight female Caucasian without a history of seizures or risk factors for atherosclerosis and DVT was referred to us for further evaluation 4 weeks after an acute-onset rightsided sensorimotor hemiparesis, dizziness and mild mnestic dysfunction. The symptoms purportedly persisted for about $12 \mathrm{~h}$ (NIH stroke scale of approx. 4) and disappeared over a period of $24 \mathrm{~h}$ without sequelae. She had a similar, but much shorter and milder episode affecting the left extremities several weeks earlier, which had been attributed to a migraine, but may also have been a transient ischemic attack (TIA). On examination, no pathological findings were detected (NIH stroke scale $=0$ ). In particular, heart murmurs and lower limb swellings were absent. Blood pressure and blood glucose on admission were $125 / 65 \mathrm{~mm} \mathrm{Hg}$ and $105 \mathrm{mg} / \mathrm{dl}$, respectively. Blood pressure monitoring during admission showed normal values.

Magnetic resonance imaging revealed a left-sided, wedge-shaped lesion in the inferotemporal cortex (fig. 1:a), clearly suggesting recent ischemia due to occlusion of a temporal branch of the posterior cerebral artery. There was neither cerebral hemorrhage nor evidence of atherosclerosis of the brain arteries. Laboratory tests including cholesterol and coagulation parameters (proteins $\mathrm{C}$ and $\mathrm{S}$, antithrombin III, prothrombin mutation and antiphospholipid antibodies) revealed no pathological findings. Cerebrospinal fluid analysis showed no evidence of cerebral inflammation. 24-hour Holter ECG showed no evidence of atrial fibrillation. Interestingly, transesophageal contrast-enhanced echocardiography (TEE + Contrast) revealed a significant PFO (fig. 1b) with spontaneous right-to-left shunt and a hypermobile interatrial septum with a maximum deviation of $8 \mathrm{~mm}$ towards the right atrium, but no evidence of intracardiac thrombus or aortic plaques. Duplex ultrasound of the lower extremities revealed no evidence of DVT.

The guidelines recommend acetylsalicylic acid 100-300 $\mathrm{mg}$ for first time CS in the setting of PFO and warfarin after a recurrence. The possible episode of TIA several weeks earlier made the current case, affecting a different territory as the former, a recurrence which made us initiate therapy with a vitamin $\mathrm{K}$ antagonist (phenprocoumon) to reach an INR between 2.0 and 3.0 as a bridge to PFO closure since the patient could not be convinced to stay on this therapy for 2 or more years as suggested in the guidelines. Twelve weeks later, an interventional fluoroscopic and TEE-guided PFO closure using an Amplatzer 25-mm device was carried out, which necessitated a dual antiplatelet therapy for 6 months. During this procedure an angiography of the brachiocephalic vein revealed residue of a venous wall adherent thrombus (fig. 1c). Anticoagulation was discontinued because the thrombus was deemed to be old and well organized. Follow-up at 3 months was uneventful. A detailed history and physical examination followed by a plain chest X-ray in 2 plains, an abdominal ultrasound and a gynecological examination including mammography revealed no findings in support of malignancy as a possible cause of the unusually located DVT.

\section{Discussion}

PFO as a conduit for paradoxical embolism causing stroke is debated, despite the existence of extensive epidemiological evidence suggesting a causal relationship between $\mathrm{PFO}$ and CS $[3,6-8]$. The prevalence of PFO among young ischemic stroke patients is 
twice as high as that of the normal population (25\%) [3]. However, PFO alone cannot represent the direct etiology of a stroke, as it always requires the presence of a thrombotic source. Here, we hypothesized paradoxical embolism as a plausible cause of the ischemic event. Paradoxical embolism is easily demonstrable on contrast echocardiography in the presence of PFO and there have been cases of transit thrombus literally 'caught in the act' of crossing the interatrial septum via a PFO [9]. Nevertheless, large trials (including the more recent CLOSURE I trial) have not proven PFO closure to be superior to other forms of therapy, such as aspirin and warfarin, in preventing recurrent CS. These studies have, however, enrolled a heterogeneous group of patients (those in which the PFO is possibly just an epiphenomenon and those with a strong evidence of paradoxical embolism) [10] coupled with the development of multiple possible causes (left atrial clot, atrial fibrillation, etc.) of recurrent stroke in the course of a trial, undermining the conclusiveness of the data interpretation. This latter reason, coupled with the fact that nearly 30,000 young patients yearly have a CS and PFO, may suggest to rethink future trial strategies [11], for example PFO closure plus best medical therapy versus best medical therapy alone. However, the uniqueness in our case is the occurrence of an inferotemporal cortex stroke following a possible TIA several weeks earlier in association with PFO in the presence of an extremely rare DVT location. This strongly suggests paradoxical embolism as the cause of the ischemic insult, especially in the absence of a cardioembolic source and atherosclerotic risk factors.

Stroke is a potentially debilitating disease with a high propensity to recur, especially in the absence of adequate therapy. Therefore, patients with DVT and a higher probability of paradoxical embolism as in the case of coexisting PFO with right-to-left shunt and stroke should, even in the absence of inherited coagulation disorders, be treated with vitamin $\mathrm{K}$ antagonist for an average period of 6 months (in keeping with DVT guidelines), followed by PFO closure. Critics rightly propose that underlying DVT is only seldom found in patients with ischemic stroke and PFO. However, this may also be due to the dissolution of the source of embolism at the time of the diagnostic investigation, the heterogeneous expertise of the sonographers or the search for such culprit DVT being limited to the lower extremities. In addition, studies on pulmonary embolism (PE) have shown a DVT prevalence of only $18 \%$ and of $36-45 \%$ in patients with suspected and with confirmed PE, respectively [12], suggesting that DVT is a frequent cause of embolism, and that the presence of PFO in these patients may be a risk factor for stroke. Furthermore, Otten et al. [13] demonstrated the existence of DVT in unusual locations in patients admitted to hospital for other reasons, suggesting that other venous sources of emboli are often overlooked. Brachiocephalic DVT is a rare entity $(<0.06 \%$ of hospitalized patients), associated with central venous catheters, Crohn's disease and occult malignancy (Trousseau's syndrome) [13-15]. A search beyond the lower limbs may become, however, necessary in case of recurrent cryptogenic stroke, especially when an embolic pattern and one or the other risk factor (e.g. central venous line, inflammatory bowel disease, malignancy, etc.) for upper limb DVT are evident.

To our knowledge, this is the first reported case of brachiocephalic DVT associated with ischemic stroke and PFO. This suggests that in selected cases such as in young patients with stroke of embolic pattern, prior central venous catheter and history of Crohn's disease or malignancy, further examination of the venous system beyond the lower extremities may be warranted. With the exclusion of cases where the thrombus has already dissolved at the time of examination, this would further strengthen the evidence for paradoxical embolism. 


\section{Acknowledgement}

We are grateful to the patient for the permission to publish this information.

\section{Disclosure Statement}

The authors have nothing to disclose.
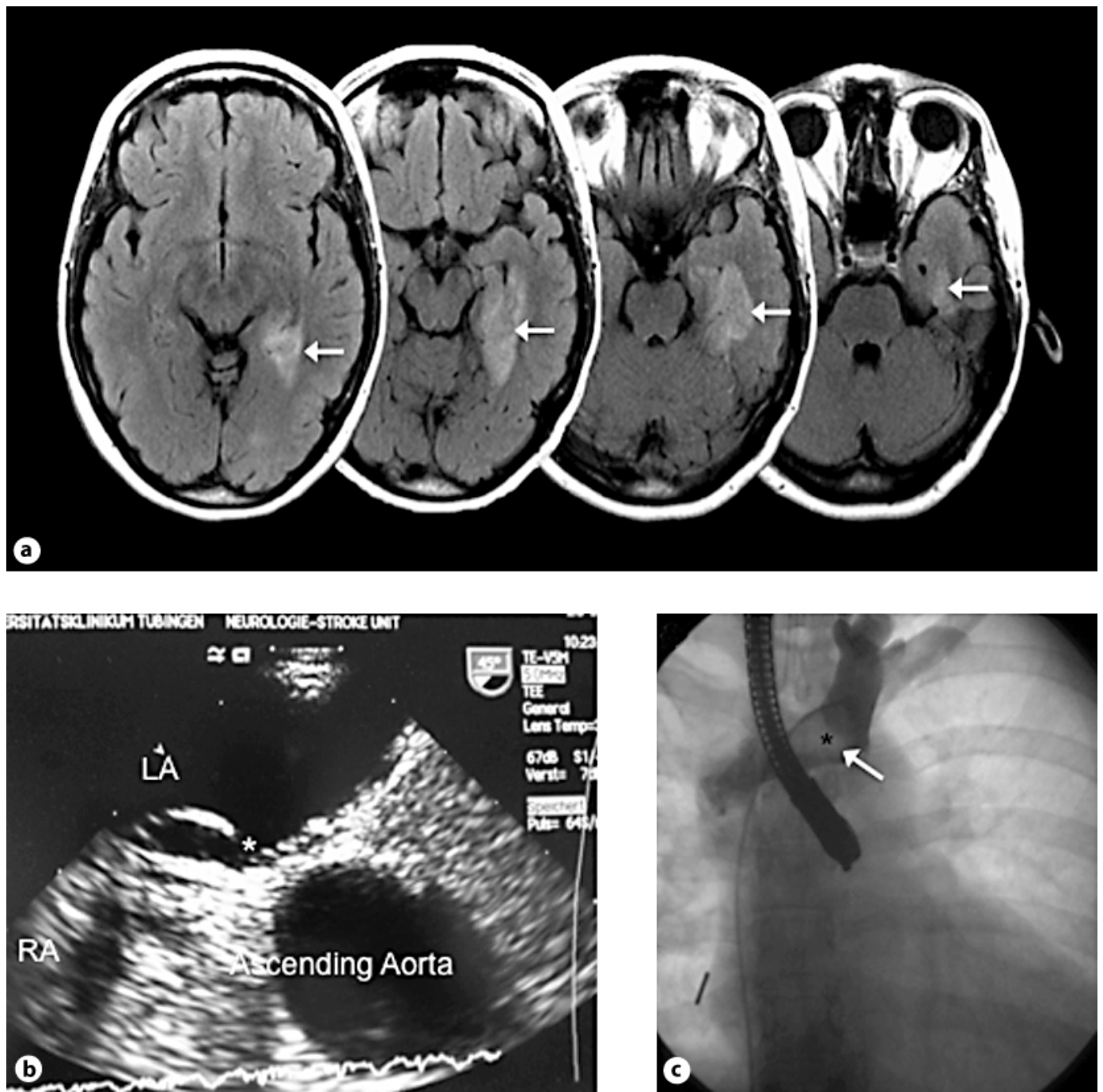

Fig. 1. a cMRT FLAIR sequence showing a $2.5 \times 1.4-\mathrm{cm}$ wedge-shaped, left-sided hyperintense lesion in the interotemporal cortex (arrows), clearly suggesting a recent ischemia due to occlusion of a temporal branch of the posterior cerebral artery. b Contrast TEE demonstrates PFO - a clear separation of the membranes of the interatrial septum (asterisk) between contrasted right atrium (RA) and contrast-free left atrium (LA). c Thoracic fluoroscopy with left brachiocephalic venography demonstrates a $2.5 \times 1.5$ $\mathrm{cm}$, wedge-shaped, superior wall adherent, radio-opaque structure, clearly suggesting a brachiocephalic vein thrombus (white arrow and black asterisk). 


\section{References}

1 Lee BI, Nam HS, Heo JH, Kim DI: Yonsei stroke registry. Analysis of 1,000 patients with acute cerebral infarctions. Cerebrovasc Dis 2001;12:145-151.

-2 Sacco RL, Ellenberg JH, Mohr JP, Tatemichi TK, Hier DB, Price TR, Wolf PA: Infarcts of undetermined cause: the NINCDS stroke data bank. Ann Neurol 1989;25:382-390.

- 3 Overell JR, Bone I, Lees KR: Interatrial septal abnormalities and stroke: a meta-analysis of case-control studies. Neurology 2000;55:1172-1179.

4 Ranoux D, Cohen A, Cabanes L, Amarenco P, Bousser MG, Mas JL: Patent foramen ovale: is stroke due to paradoxical embolism? Stroke 1993;24:31-34.

5 Johnson BI: Paradoxical embolism. J Clin Pathol 1951;4:316-332.

6 Windecker S, Wahl A, Nedeltchev K, Arnold M, Schwerzmann M, Seiler C, Mattle HP, Meier B: Comparison of medical treatment with percutaneous closure of patent foramen ovale in patients with cryptogenic stroke. J Am Coll Cardiol 2004;44:750-758.

7 Rodes-Cabau J, Noel M, Marrero A, Rivest D, Mackey A, Houde C, Bedard E, Larose E, Verreault S, Peticlerc M, Pibarot P, Bogaty P, Bertrand OF: Atherosclerotic burden findings in young cryptogenic stroke patients with and without a patent foramen ovale. Stroke 2009;40:419-425.

-8 Ozdemir AO, Tamayo A, Munoz C, Dias B, Spence JD: Cryptogenic stroke and patent foramen ovale: clinical clues to paradoxical embolism. J Neurol Sci 2008;275:121-127.

9 Chow V, Wang W, Wilson M, Yiannikas J: Thrombus in transit within a patent foramen ovale: an argument for consideration of prophylactic closure? J Clin Ultrasound 2011, DOI: 10.1002/jcu.20820.

10 Alsheikh-Ali AA, Thaler DE, Kent DM: Patent foramen ovale in cryptogenic stroke: Incidental or pathogenic? Stroke 2009;40:2349-2355.

11 Thaler DE, Kent DM: Rethinking trial strategies for stroke and patent foramen ovale. Curr Opin Neurol 2010;23:73-78.

12 van Rossum AB, van Houwelingen HC, Kieft GJ, Pattynama PM: Prevalence of deep vein thrombosis in suspected and proven pulmonary embolism: a meta-analysis. Br J Radiol 1998;71:1260-1265.

13 Otten TR, Stein PD, Patel KC, Mustafa S, Silbergleit A: Thromboembolic disease involving the superior vena cava and brachiocephalic veins. Chest 2003;123:809-812.

14 Kimura T, Chino M, Ogasawara N, Nakano T, Izumi S, Takeuchi H: Trousseau's syndrome with brachiocephalic vein thrombosis in a patient with uterine carcinosarcoma. A case report. Angiology 1999;50:515-518.

15 Ribeiro JM, Rebocho L, Lucas MB, Campos P, Victorino RM: Brachiocephalic vein thrombosis associated with Crohn's disease. J Gastroenterol 2003;38:268-271. 